\title{
RENAL TUBERCULOSIS IN A MIDDLE AGED WOMAN PRESENTING WITH ASYMPTOMATIC MICROSCOPIC HAEMATURIA AND ERYTHEMA NODOSUM
}

\author{
ABDUL WADUD CHOWDHURY, ${ }^{1}$ MUHAMMAD RAFIQUL ALAM, ${ }^{2}$ AMITAV SAHA, ${ }^{3}$ MOHAMMAD \\ MAHBUB JAMIL ${ }^{4}$
}

\begin{abstract}
:
Erythema nodosum is a common association of tuberculosis (TB), especially in this part of the world. Urogenital TB, although less common than other form of tuberculosis, may be associated with erythema nodosum along with other urinary complaints. Here we present a case of an old lady who presented with erythema nodosum with painless haematuria (microscopic) which was later found to be of tuberculous aetiology. Urine analysis yielded no acid-fast bacilli but culture on special media showed growth of Mycobacterium tuberculosis. Though erythema nodosum is usually associated with primary $T B$, our case revealed it can be found in cases where pulmonary focus is not the primary origin. The patient responded well to anti-tubercular drugs and is doing well on follow-up.
\end{abstract}

Keywords: Erythema nodosum, Renal Tuberculosis, Urogenital Tuberculosis.

\section{Introduction :}

Erythema nodosum (EN) is the most common type of panniculitis. ${ }^{1}$ Review of literature shows that the etiology varies in frequency between different regions of the world. Among the etiologies of EN, tuberculosis (TB) is the most frequent disorder in developing countries. ${ }^{2,3}$ TB can have many clinical-pathological patterns. From the pulmonary focus, 2 to $20 \%$ of the patients go on to develop urogenital TB, ${ }^{4}$ which is a form of secondary TB. EN is seen only in the primary TB form of tuberculous diseases. ${ }^{2,3}$ Here we report a case of renal TB which was diagnosed while investigating a case of $\mathrm{EN}$.

\section{Case Report :}

A 47 years old lady presented with multiple palpable, tender, non itchy, non-blanching reddish swelling of variable size and shape on both forearms and legs for the last three years. She is hypertensive with a strong family history of hypertension and ischemic heart disease (IHD) and has been suffering from effort angina (class II) for five years. She was on medication under regular supervision of a cardiologist and was adequately managed. For her presenting complaint, she had already visited several dermatologists who diagnosed the case as EN. She was treated with oral potassium iodide (KI) solution, local application of salicylic acid in methanol and glycerin and some steroid creams. These measures conferred only limited improvement. Investigation records show a highly positive Tuberculin skin test $(17 \mathrm{~mm})$ and $2-4 \mathrm{RBC} /$ $\mathrm{HPF}$ on urine R/M/E at that time.

At the time of presentation to our consultation, she had florid lesions which we also diagnosed as EN. She had no history of recent sore throat, fever, weight loss, cough or urinary symptoms. She was not having any antibiotics or oral contraceptives. She was not pregnant. There were no symptoms suggestive of chronic bowel disease. Chest X-ray P/A view showed no hilar adenopathy or evidence of any recent or past infection. General and systemic examinations were normal except for the EN. No biopsy was taken as the diagnosis was clinically obvious.

However, a Tuberculin skin test was again highly positive at $19 \mathrm{~mm}$. There was also microscopic

1. Associate Professor, Department of Cardiology, Dhaka Medical College, Dhaka.

2. Professor, Department of Nephrology, Bangabandhu Sheikh Mujib Medical University, Dhaka.

3. Post graduate trainee, Department of Medicine, Dhaka Medical College, Dhaka.

4. General practitioner, Dhaka.

Correspondence : Dr. Abdul Wadud Chowdhury, Associate Professor, Department of Cardiology, Dhaka Medical College, Dhaka, Email : drwadud@hotmail.com 
haematuria (20-30 RBC / HPF) on several consecutive samples but no pus cell or any cast. Culture on routine media showed growth of S. saprophyticus and the patient was started on antibiotics according to the sensitivity report. Repeat culture showed no growth, but haematuria was still present. Intravenous urogram (IVU) and abdominal ultrasonogram (USG) were normal, making the diagnosis of neoplastic lesions or stone disease highly unlikely. Other investigations including random blood sugar \& S. creatinine were within normal limits. RA titre and ANA were negative. ECG and echocardiogram showed features of IHD. Hb was $11 \mathrm{gm} / \mathrm{dL}$, ESR $30 \mathrm{~mm}$ in the first hour with normal white cell count, distribution and normal platelet count \& morphology. Three consecutive morning samples of urine failed to show any organism on acid fast bacilli staining. However, on culture, three similar samples grew Mycobacterium tuberculosis on special media.

In light of the fact that Mycobacterium tuberculosis was cultured from the urine while IVU and USG did not pick up any identifiable bladder or ureteric lesion, a diagnosis of renal TB was made. The patient was started on anti TB drugs with standard four drugs regimen without steroid. It was noticed that, the EN started resolving soon after the initiation of treatment and regressed to only faint macular staining at the end of two months.

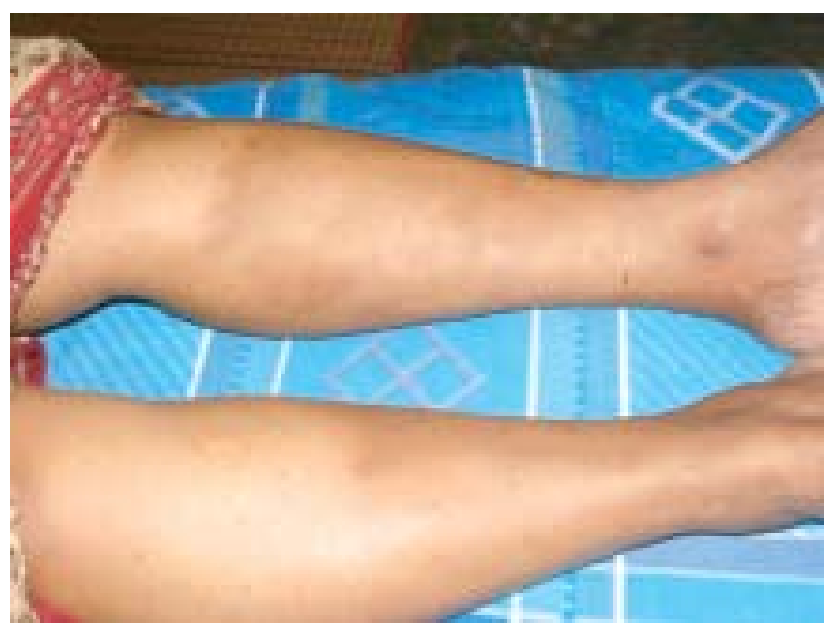

Fig.-1: The macular lesions suggest healed EN. Note that the lesions are healing without any residual scarring

The patient is now in the continuation phase of the treatment and on regular follow up with improvement of haematuria.

\section{Discussion :}

$\mathrm{EN}$ is the commonest form of panniculitis that is characterized by a neutrophilic infiltrate of the septa dividing the subcutaneous fat. EN is not associated with vasculitis, although small vessel inflammation and hemorrhage can occur. It appears as painful, symmetric, red nodules most commonly on the anterior legs but can also appear in the forearms. Lesions involute in weeks with bruise-like appearance without ulceration. They tend to heal completely. ${ }^{1}$ Although a specific cause has never been identified, there are several inciting triggers. In a developed country like the United States, upto 55\% were idiopathic. ${ }^{1}$ Streptococcal pharyngitis along with other infections like mycoplasma, chlamydia, histoplasmosis, coccidioidomycosis, mycobacteria accounted for $28 \%$ to $48 \%$ of the cases. ${ }^{1}$ Other causes included Sarcoidosis (11\% to $25 \%$ ) with bilateral hilar adenopathy, drugs like oral contraceptives, antibiotics e.g. sulfonamides, amoxicillin ( $3 \%$ to $10 \%$ ), pregnancy ( $2 \%$ to $5 \%$ ), enteropathies like regional enteritis, ulcerative colitis ( $1 \%$ to $4 \%$ ). Some rare causes constituted for less than $1 \%$ of cases. ${ }^{1}$ In the developing countries however, TB remains the most frequent cause. ${ }^{2}$ A prospective study in south India showed that TB was the major culprit. ${ }^{5}$ Streptococcal infections and sarcoidosis are the commonest cause of EN in western literatures, $6,7,8$ the Indian study had only one case with preceding pharyngitis and raised ASO titre, which responded to antibiotics. A prospective study in Turkey also showed primary TB as the main etiology. ${ }^{9}$ In the mid twentieth century, even some of the now developed countries reported primary TB as the main cause of EN ${ }^{3}$ However, EN associated with TB was only described in the context of primary TB. ${ }^{2,3}$ In our case report, we tried to associate a secondary form of TB (renal TB) with EN.

The diagnosis of EN is clinical with only doubtful cases requiring a biopsy. ${ }^{1}$ In the described case, diagnosis was clinically obvious. The lesions can be confused with erythema induratum (EI) of Bazin, but EI tends to ulcerate and leave residual scarring. ${ }^{10} \mathrm{~A}$ case of EI in association with renal TB has been described. ${ }^{11}$ The EN in our patient did not ulcerate and regressed with only macular patches remaining at this point. In a retrospective study in Switzerland $77 \%$ of infection-induced EN healed after 7 weeks, the longest course being 18 weeks. ${ }^{12}$ This virtually excludes the infectious causes like streptococcus, mycoplasma, chlamydia, histoplasmosis, coccidioidomycosis in our 
case, as they are not likely to cause prolonged symptoms. Idiopathic EN also tends to cause prolonged disease. In the Swiss study mentioned above, $30 \%$ of idiopathic EN lasted more than 6 months. ${ }^{12}$ Patients in whom EN was associated with non-Hodgkin lymphoma also had an extremely protracted course. ${ }^{12}$ But none of these explain the prompt resolution of EN after starting anti TB drugs, especially when treatment was initiated without steroids. This leaves our diagnosis of renal TB as the only probable association.

The most common symptoms of renal TB at presentation are storage symptoms (urgency, frequency), dysuria, and hematuria, affecting $50.5 \%$, $37.9 \%$, and $35.6 \%$ of cases, respectively. ${ }^{13}$ In another study in Brazil, $7.5 \%$ of the cases had no lesions or there were minimal lesions on radiographic examination. TB was diagnosed when bacilli were shown in the urine. ${ }^{4}$

A study was conducted in India to establish the efficacy of antitubercular therapy in patients with idiopathic EN with a highly positive Mantoux test (average 19 $\mathrm{mm}$ ). It was concluded that, antitubercular therapy definitely has a role in the management of patients with EN, even in the absence of a detectable focus of tubercular infection, especially in regions where TB is endemic. ${ }^{14}$ Therefore, we can conclude that, TB should be high in the list of probable causes when investigating EN and asymptomatic haematuria.

\section{Conclusion :}

According to the WHO report of 2009 on 'Global Tuberculosis Control, epidemiology, strategy, financing', Bangladesh ranks 6th on the list of 22 highest burden TB countries in the world. Due to this heavy burden, many different presentations of TB can be seen by the clinician. Early diagnosis and treatment is very crucial, not only for the life of that individual patient, but also for containment of the disease for benefit of the community. It is critical to have an open mind when the diagnosis of TB is on the table. The reported case is a reminder of the association between TB and EN. At the same time, it should serve to break an age-old dogma regarding appearance of EN only in cases of primary TB.

\section{References :}

1. Schwartz RA, Nervi SJ. Erythema nodosum: a sign of systemic disease. Am Fam Physician 2007; 75(5): 695-700.
2. Mert A, Ozaras R, Tabak F, Ozturk R. Primary tuberculosis cases presenting with erythema nodosum. J Dermatol 2004; 31(1): 66-8.

3. Doxiadis SA. Aetiology of erythema nodosum in children. Br Med J 1949 ;2(4632):844.

4. Figueiredo AA, Lucon AM, Gomes CM, Srougi M. Urogenital tuberculosis: patient classification in seven different groups according to clinical and radiological presentation. Int Braz J Urol 2008; 34(4): 422-32.

5. Sarveswari KN, Swamikannu M, Ramakrishnan S. An aetiological analysis of erythema nodosum in a community hospital. Indian J Dermatol Venereol Leprol 2001; 67(6): 287-9.

6. Cribier B, Caille A, Heid E, Grosshans E. Erythema nodosum and associated diseases: A study of 129 cases. Int J Dermatol 1998; 37(9): 667-72.

7. García-Porrúa C, González-Gay MA, VázquezCaruncho M, et al. Erythema nodosum: etiologic and predictive factors in a defined population. Arthritis Rheum 2000; 43(3): 584-92.

8. Psychos DN, Voulgari PV, Skopouli FN, Drosos AA, Moutsopoulos HM. Erythema nodosum: the underlying conditions. Clin Rheumatol 2000; 19(3): 212-6.

9. Mert A, Ozaras R, Tabak F, Pekmezci S, Demirkesen C, Ozturk R. Erythema nodosum: an experience of 10 years. Scand J Infect Dis 2004; 36(6-7): 424-7.

10. Murphy GF, Mihm MC. The skin.In:Kumar V,Abbas AK, Fausto N. Robbins and Cortan, eds. Pathologic basis of disesase, $7^{\text {th }}$ ed. Philadelphia: Saunders, 2004: 1227-72.

11. Daher Ede F, Silva Júnior GB, Pinheiro HC, Oliveira TR, Vilar Mdo L, Alcântara KJ. Erythema induratum of Bazin and renal tuberculosis: report of an association. Rev Inst Med Trop Sao Paulo 2004; 46(5): 295-8.

12. Bohn S, Büchner S, Itin P. Erythema nodosum: 112 cases. Epidemiology, clinical aspects and histopathology. Schweiz Med Wochenschr 1997; 127(27-28): 1168-76.

13. Figueiredo AA, Lucon AM. Urogenital tuberculosis: update and review of 8961 cases from the world literature. Rev Urol 2008; 10(3): 207-17.

14. Kumar B, Sandhu K. Erythema nodosum and antitubercular therapy. J Dermatolog Treat 2004; 15(4): 218-21. 